\title{
General Psychiatry Biomarkers for the diagnosis of Alzheimer's disease, dementia Lewy body, frontotemporal dementia and vascular dementia
}

Joshua Marvin Anthony Maclin, ${ }^{1,2,3}$ Tao Wang, ${ }^{2,3}$ Shifu Xiao ${ }^{2,3}$

To cite: Maclin JMA, Wang T, Xiao S. Biomarkers for the diagnosis of Alzheimer's disease, dementia Lewy body, frontotemporal dementia and vascular dementia. General Psychiatry 2019;32:e0054. doi:10.1136/ gpsych-2019-100054

Received 23 August 2018 Revised 14 January 2019 Accepted 15 January 2019
Check for updates

(C) Author(s) (or their employer(s)) 2019. Re-use permitted under CC BY-NC. No commercial re-use. See rights and permissions. Published by BMJ.

${ }^{1}$ Department of Geriatric Psychiatry, Shanghai Mental Health Center, Shanghai Jiao Tong University School of Medicine, Shanghai, China

${ }^{2}$ Alzheimer's Disease and Related Disorders Center, Shanghai Jiao Tong University, Shanghai, China

${ }^{3}$ Department of Neuroscience, Earlham College, Richmond, Indiana, USA

Correspondence to Professor Tao Wang; wtshhwy@163.com

\section{ABSTRACT}

Background Dementia is a chronic brain disorder classified by four distinct diseases that impact cognition and mental degeneration. Each subgroup exhibits similar brain deficiencies and mutations. This review will focus on four dementia subgroups: Alzheimer's disease, vascular dementia, frontotemporal dementia and dementia Lewy body.

Aim The aim of this systematic review is to create a concise overview of unique similarities within dementia used to locate and identify new biomarker methods in diagnosing dementia.

Methods 123300 articles published after 2010 were identified from PubMed, JSTOR, WorldCat Online Computer Library and PALNI (Private Academic Library Network of Indiana) using the following search items (in title or abstract): 'Neurodegenerative Diseases' OR 'Biomarkers' OR 'Alzheimer's Disease' OR 'Frontal Temporal Lobe Dementia' OR 'Vascular Dementia' OR 'Dementia Lewy Body' OR 'Cerebral Spinal Fluid' OR 'Mental Cognitive Impairment'. 47 studies were included in the qualitative synthesis.

Results Evidence suggested neuroimaging with amyloid positron emission tomography (PET) scanning and newly found PET tracers to be more effective in diagnosing Alzheimer's and amnesiac mental cognitive impairment than carbon-11 Pittsburgh compound-B radioisotope tracer. Newly created methods to make PET scans more accurate and practical in clinical settings signify a major shift in diagnosing dementia and neurodegenerative diseases.

Conclusion Vast improvements in neuroimaging techniques have led to newly discovered biomarkers and diagnostics. Neuroimaging with amyloid PET scanning surpasses what had been considered the dominant method of neuroimaging and MRI. Newly created methods to make PET scans more accurate and practical in clinical settings signify a major shift in diagnosing dementia pathology. Continued research and studies must be conducted to improve current findings and streamline methods to further subcategorise neurodegenerative disorders and diagnosis.

\section{INTRODUCTION}

The four types of dementia represent a destructive, prevalent and debilitating mental disorder affecting millions worldwide. To accurately diagnose the disorder, much neurophysiological and lab testing must be conducted. However, difficulties arise when similarities in characteristic readings are inconclusively interpreted to distinguish between the four types of dementia, thus the need for continued identification, through research, of new biomarker testing apart from cerebrospinal fluid (CSF) biomarker testing. ${ }^{1-3}$ CSF has accounted for the discovery of most known biomarkers specific to dementia. The vast majority of biomarkers are only obtained through lumbar punctures. The results that come from these methods are heavily dependent on the age and physical state of the patient, which makes this method ineffective despite it being the main method for diagnosing dementia. There is common aetiology and significant overlapping of biomarkers between dementia types, which make them harder to diagnose. For instance, overlapping concentrations occur in CSF total tau (T-tau) and tau phosphorylated at threonine 181 (P-tau181P) between Alzheimer's disease $(\mathrm{AD})$, frontotemporal dementia (FTD), dementia Lewy body (DLB) and vascular dementia $(\mathrm{VaD}){ }^{1}{ }^{4}$ Developing less invasive methods used to evaluate dementia biomarkers, specific to a certain type of dementia, is one of the main objectives of recent neurodegenerative research. Employing alternative methods to diagnose different types of dementia will require advancements in neuroimaging techniques such as positron emission tomography (PET) scans and MRIs.

There are two types of biomarkers, exposure (risk prediction) and disease (screening and diagnosis), which are generally defined as cellular, biochemical or molecular alterations that are measured in human tissues or fluids. ${ }^{1-6}$ The use of biomarkers is specific 
to dementia and can be used to diagnose and manage cardiovascular disease, immunological and genetic disorders. ${ }^{56}$ The wide uses for biomarkers and the advancements of such will provide much-needed clarity and understanding of the wide range of neurological diseases.

$\mathrm{AD}$ is characterised by the gradual loss of memory, cognition and activities of daily life (ADL). AD is the most wellknown and prevalent type of dementia in the world, with a $69 \%$ occurrence rate in patients older than 75 years of age. ${ }^{7} \mathrm{AD}$ is also responsible for plaque deposit build-up of amyloid beta (Ab1-42), T-tau and P-tau181P. These four biomarkers represent the most clinically tested measurements for diagnosing dementia disorder. Despite heavy utilisation of CSF biomarkers in clinical trials throughout the diagnostic process, biomarkers often lose diagnostic performance attributes due to patient ageing that are needed to accurately differentiate between $\mathrm{AD}$ and $\mathrm{DLB}$, or any other comparative form of dementia. ${ }^{489}$

DLB and AD share similar aetiologies, making conclusive diagnosis harder to ascertain, therefore increasing the likelihood for misdiagnosis. Neuropsychosocial profiles observed exhibiting cross-sectional comparisons in mild or early stages of $\mathrm{AD}$ requires biomarker measurements specific to each disorder to render more conclusive results.

Degradation of the temporal and frontal lobes is the root cause of behavioural and physical maladies specific to FTD, a heterogeneous disease diagnosed by evaluating a patient's family history. Given that FTD is the second most common type of dementia for those under the age of 65 , it is the easiest subtype to conclusively diagnose. ${ }^{10-12}$ The chronic degradation of the frontal and temporal lobes eventually distorts one's behavioural personality by diminishing the ability to perform social and executive functions needed for coherent communication. ${ }^{13} 14$ The myriad of maladies results in two subtypes: the behavioural subtype, which accounts for half of patients with FTD, and the language subtype, which has three other variants. ${ }^{1011}{ }^{14}$ Language variants are referred to as variants of primary progressive aphasia (PPA), semantic variants of primary progressive aphasia (svPPA) and non-fluent variant of primary progressive aphasia (nfvPPA). ${ }^{1011}$

$\mathrm{VaD}$ is a heterogeneous disease genetically related to cerebrovascular disease. The likelihood of someone developing $\mathrm{VaD}$ increases when a person has cerebrovascular disease. ${ }^{6}$ Prior to diagnosing VaD, patients must be diagnosed with mental cognitive impairment (MCI) due to an inability to perform ADL. ${ }^{15}$ If higher levels of cerebrovascular lesions are identified during initial testing, vascular ischaemia becomes the main determining factor for diagnosing a patient with vascular mild cognitive impairment (vMCI). If symptoms worsen, steps are taken to diagnose the patient with $\mathrm{VaD} .{ }^{1516}$

\section{METHODS}

Data were sourced from review journals and clinical studies via PubMed. The following are the supplemental databases sourced on 28 June 2018: PALNI (Private Academic Library Network of Indiana), JSTOR (journals, primary sources and books) and WorldCat Online Computer Library. PubMed and PALNI were sourced on 2 July 2018. The review protocol may be accessed at https://gpsych.bmj.com/pages/authors/. All articles referenced in this systematic review are categorised by publication date, article content and evidence-based research. There were 123300 articles published after 2010 identified from the databases using the following search items (in title or abstract): 'Neurodegenerative Diseases' OR 'Biomarkers' OR 'Alzheimer's Disease' OR 'Frontal Temporal Lobe Dementia' OR 'Vascular Dementia' OR 'Dementia Lewy Body' OR 'Cerebral Spinal Fluid' OR 'Mental Cognitive Impairment'. In total, 57 studies were researched for this review. Forty-seven studies were used in the analysis. Articles displaying publication bias were eliminated from the review. Articles chosen referenced advancements in genetics, neuroimaging and the localisation of new biomarkers that indicate specific types of dementia. There were other articles dating back to 1992 that were used for background purposes (figure 1).

\section{RESULTS}

\section{Current biomarkers and the implementation of blood plasma} and serum biomarkers

Obtaining and testing CSF through lumbar punctures result in intense pain for patients and provide little clarity in differentiating types of dementia. ${ }^{125}$ Lumbar punctures may cause severe backaches, nausea and fatigue in subjects most afflicted. This painful and invasive method used to analyse CSF often renders inconclusive results and is not considered a major biomarker for diagnosing dementia. It fails to identify a specific type of dementia with precision. ${ }^{1} 2517$ Indepth research into serum, plasma and microRNA (miRNA) dysregulation has laid groundwork for more effective testing in the diagnostic process. ${ }^{1718}$ The use of miRNA dysregulation appears to be the most effective new method developed for use in diagnosing the four different types of dementia. Further evaluation of the dysregulation of miRNA expression in peripheral blood may serve as a potent source for diagnosing $\mathrm{AD}$ and other neurodegenerative diseases. ${ }^{17}{ }^{18}$ Most importantly, this process delivers a less painful experience for the patient, which prompts frequent testing and better follow-up evaluations. In relation to $\mathrm{AD}$, a study was conducted showing the effectiveness of this method by identifying a plethora of downregulated miRNAs using microarray chips that compared 16 patients with $\mathrm{AD}$ with 16 control patients. ${ }^{17}$ The downregulated miRNAs (miR$34 a$, miR-81b and let-7f) were a part of p53, Notch and Bcl-2 pathways found in $\mathrm{AD}$ pathogenesis. ${ }^{17}$

There are a multitude of factors involved in $\mathrm{AD}$ degeneration and neurodegeneration. The primary factors resulting from mitochondrial ultrastructural failure include a large formation of hypoxic mitochondria, overproduction of mitochondrial DNA deletions, and/or the 


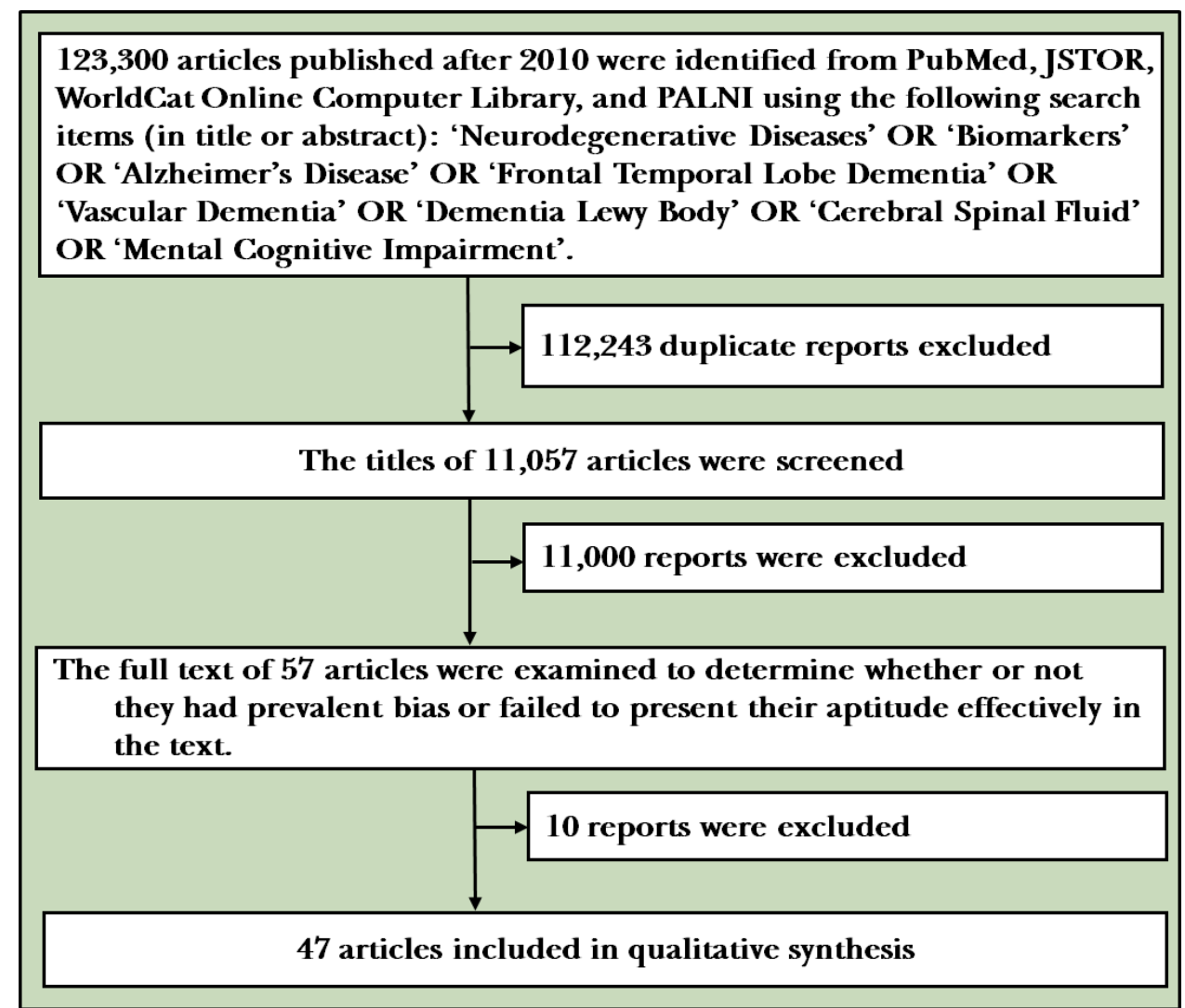

Figure 1 Flowchart of collecting and evaluating research used in this review. PALNI, Private Academic Library Network of Indiana.

build-up of neurofibrillary tangles plaque and amyloid beta plaque. ${ }^{19-21}$ Mitochondrial failure is the primary factor for hypoperfusion caused by chronic injury from decreased blood flow to the brain. This may lead to other degenerative symptoms such as oxidative stress and neuronal damage. ${ }^{20}{ }^{22}$ This mitochondrial failure is induced by the vascular endothelial cells, neurons and the glia cells' inability to synthesise, store and release active oxygen (ROS) and vasoactive substances in response to stimuli, especially when formed by chronic hypoperfusion. ${ }^{19} 2324$ Apart from the various possible side effects of mitochondrial failure, diagnosing $\mathrm{AD}$ is a combination of neurological examination, brain imaging and mental status tests in conjunction with the use of biomarkers currently used to diagnose patients with $\mathrm{AD} .^{21}$

DLB is arguably one of the hardest types of dementia to identify having an unclear pathology due to lack of known biomarkers for DLB and its similar aetiology with $\mathrm{AD}$. Additional investigation is needed to distinguish DLB from $\mathrm{AD}$ by using blood-based biomarkers in clinical settings. However, if blood-based testing became a method of biomarker collection, inaccurate reflections of disease progression could result, as blood is not in direct contact with the central nervous system. ${ }^{425}$ In an effort to differentiate $\mathrm{AD}$ from $\mathrm{DLB}$, a study was conducted with patients diagnosed with AD, FTD, DLB and Parkinson's disease (PD). The researchers assumed the addition of CSF 3-methoxy-4-hydrox-yphenylglycol (MHPG), a main metabolite of monoamines epinephrine and norepinephrine that aids in identifying central noradrenergic activity, would add discriminative value that would increase both sensitivity and specificity for differentiating between $\mathrm{AD}$ and DLB. ${ }^{4}$ These diseases were compared using ELISA to measure amyloid beta, T-tau and tau (phosphorylated at threonine 181) levels. ${ }^{4}$ Moreover, the study resulted in MHPG levels being much higher in the baseline levels, while serum MHPG levels were lower in patients with DLB compared with all of the other groups. ${ }^{4}$ Solely focusing on MHPG levels in CSF and serum monoamines, investigators found that CSF and serum MHPG were the most valuable markers to differentiate $\mathrm{AD}$ from both $\mathrm{DLB}$ and PD. ${ }^{4}$

Eicosapentaenoic acid (EPA) and docosahexaenoic acid (DHA) are plasma fatty acids that are part of the omega-3 polyunsaturated fatty acids class ( $\omega-3$ PUFAs $).^{7}$ EPA, DHA and omega-3 have been shown to help negate the inflammatory tendencies in neurodegenerative disorders. $^{7826}$ Chronic inflammation is one of the leading causes for cerebrovascular disease. More specifically, these biomarkers were shown to improve cognitive ability for episodic memory, learning functions in healthy adults and cellular function changes in gene expression, and indicate whether fatty acid balance is optimal. ${ }^{726}$ The function of $\omega-3$ PUFAs in regard to EPA and DHA was shown to inhibit triglyceride synthesis, cause vascular relaxation, reduce platelet aggregation and reduce 
inflammation. ${ }^{726}$ When $\omega-3$ PUFAs were in decline, the more at risk a person became for developing $\mathrm{AD}{ }^{7}$

Biomarkers specific to inflammation are important for the initial diagnosis of dementia. ${ }^{37}$ Lipid biomarkers are randomly used in similar diagnostics, but primarily used to assess one's risk for cerebrovascular disease, potentially leading to VaD or metabolic syndrome (MetS). MetS was labelled as a global epidemic by the WHO due to its findings of brain abnormalities and severe declines in cognitive function of those affected..$^{21}{ }^{27}$ High-density lipoprotein (HDL), low-density lipoprotein (LDL), higher sensitivity $\mathrm{C}$ reactive protein (hsCRP) and serum amyloid A (serum AA) are plasma fatty acids used to prevent neurodegenerative developments from progressing. ${ }^{7}$ After further review of plasma fatty acid biomarkers, HDL performed a wide range of functions including antioxidation, anti-inflammation, proendothelial function and modulation of immune function. Higher levels of LDL appeared to lead to atherosclerosis, a chronic disease characterised by the deposition of excessive cholesterol in the arterial intima, resulting in increased risks of heart attacks and ischaemic stroke (IS). ${ }^{27} 28$ The hsCRP testing measurement quantifies the worsening of cognitive decline after a stroke occurrence. ${ }^{7}$ Serum AA occurs in the acute phase protein and is present in atherosclerosis, $\mathrm{AD}$, inflammatory, tumour tissue and histologically normal adults. ${ }^{7}$ Reptin and adiponectin, although not a lipid specific biomarker, are extremely beneficial for physicians to measure during the initial diagnostic process. Systemic viability, antagonistic roles in tissue growth, gene transcription, remodelling of chromatin, DNA damage sensing and repair, regulation of the tumour metastasis oppressor gene, and involvement in tumour biology are all orchestrated by reptin protein. ${ }^{7}$ Likewise, adiponectin, a hormone used to detect one's risk for coronary artery disease, is only secreted through adipocytes and causes gradual digression of atherogenesis endothelial function and vascular remodelling through the modulation of signalling cascades. ${ }^{29}$ In relation to plasma fatty acids and other biomarkers mentioned in the preceding paragraph, a study was conducted to measure the varying expressions of HDL, LDL, hsCRP, serum AA, reptin and adiponectin, as well as EPA and DHA, within patients with $\mathrm{AD}, \mathrm{VaD}$, MCI and IS. Due to a sizeable population of 776 participants $(\mathrm{AD}, \mathrm{n}=295$; MCI, $\mathrm{n}=47$; VaD, $\mathrm{n}=34 ; \mathrm{IS}, \mathrm{n}=200$; and control sector [102 males and 98 females]), conclusive results were obtained. ${ }^{7}$ Serological data for EPA, DHA, adiponectin, reptin, hsCRP, serum AA, HDL and LDL were obtained from 696 participants (normal controls, $\mathrm{n}=130 ; \mathrm{AD}, \mathrm{n}=266$; MCI, $\mathrm{n}=44$; VaD, $\mathrm{n}=33$; IS, $\mathrm{n}=200$ ) using blood samples to analyse for routine biochemical parameters. ${ }^{7}$ Regarding the Bradford protein assays collected from serological testing, EPA and DHA were assayed using gas chromatography and mass spectrometry, while adiponectin, reptin and serum AA were assayed using solid-phase sandwich ELISA through commercial kits. ${ }^{7}$ The results of the serological tests revealed EPA levels were significantly lower in patients with $\mathrm{AD}$ and IS than in the control group. ${ }^{7}$ The results also showed DHA was present at noticeably lower levels than in other dementia types analysed. Patients with VaD and IS exhibited significantly higher hsCRP levels when compared with patients with $\mathrm{VaD}$, who exhibited considerably higher levels than the control group. Serum AA levels tested higher in each control group than the control group with $\mathrm{VaD}$ and MCI, which revealed markedly higher serum AA levels than in all other groups tested. Lastly, despite exhibiting higher reptin levels, IS presented with significantly lower adiponectin levels when compared with $\mathrm{AD}, \mathrm{MCI}$ and the other measured diseases. ${ }^{7}$

Testing for HDL and LDL revealed the most consistent results in control and experimental groups tested. In the experimental groups, patients with $\mathrm{AD}$ and IS tested exhibited slightly lower levels of HDL when compared with the levels measured in patients with MCI. AD and IS in contrast exhibited significantly higher LDL when compared with other control groups with IS. From the results gathered from this study, it suggests that lower EPA and DHA levels and higher reptin and LDL levels are linked to $\mathrm{AD}$ and IS. The serum AA levels were found to be strongly associated with all of the tested cognitive diseases, whereas the reptin:adiponectin ratio was found to be strongly associated with IS and hsCRP levels heavily associated with VaD and IS. ${ }^{7}$ Amyloid beta, a proteolytic product of amyloid precursor protein, was shown to be extremely important in diagnosing $\mathrm{AD}$ due to continued production and accumulation over time in the brain of those affected with $\mathrm{AD}{ }^{30}$

Amyloid beta is thought to impair Ca2+ pumps, which leads to an influx in $\mathrm{Ca} 2+$ through voltage-dependent channels and glutamate receptors. ${ }^{13}$ Annexin A5 was the $\mathrm{Ca} 2+$ protein that was most responsible for this process and was analysed in a study of AD mice model that found that the levels of annexin A5 were augmented in both the blood and brain plasma. ${ }^{13}{ }^{30}$ It should be stated that 50 lines of transgenic mice express the same human amyloid beta precursor protein, but are considered incomplete disease models, ${ }^{31}$ although the mice results may be used for studying the mechanisms for amyloid beta generation, aggregation, clearance and toxicity. ${ }^{31}$ As for the annexin A5 levels that were evaluated between the test subjects and the control, the plasma levels were much higher in AD-affected mice than in the control group, ${ }^{13}{ }^{30}$ suggesting annexin A5 may be an effective biomarker for $\mathrm{AD}$ and possibly other types of dementia. DLB specifically was proven responsive to annexin A5 as an effective biomarker. ${ }^{232}$ Due to amyloid beta-impaired Ca2+ pumps, the increased levels of annexin A5 levels were thought to be a way for the body to compensate for the Ca2+-induced damaged as annexin A5 binds both Ca2+ and lipids. ${ }^{13}$ Annexin A5 was shown to play a role in reducing the toxicity of amyloidogenic proteins, islet amyloid proteins and $\alpha$-synuclein, which impairs complex I-dependent respiration. 33 34 These benefits defend against apoptosis, a term used to categorise an organised 
collapse of a cell by membrane blebbing (a part of a cell that bulges out below the plasma membrane), cell shrinkage, condensation of chromatin and the rapid engulfment of DNA. ${ }^{35}$ Mentioned previously, $\alpha$-synuclein is one of the proteins that impair complex I-dependent respiration, but it is also an integral protein in the development of both $\mathrm{AD}$ and PD. ${ }^{634} \alpha$-Synuclein goes through protein aggregation, causing it to switch from its physiological role of improving ATP synthase to a pathological toxic gain of function. ${ }^{34}$ That pathological function through monomer aggregation generates beta sheetrich oligomers that localise to the mitochondria in close proximity to several mitochondrial proteins such as ATP synthase. ${ }^{6444}$ Apolipoprotein E (APOE) $\varepsilon 4$ allele is the most common genetic variant of late-onset $\mathrm{AD}$ and is also associated with $\mathrm{AD}$ biomarkers, greater amyloid deposition and a faster neurodegeneration rate. ${ }^{36} 37$ A study was conducted to evaluate the effectiveness of APOE $\varepsilon 4$ status on amyloid deposition, neurodegeneration and cognition in patients with early metal cognitive impairment, the earliest stage of prodromal AD. ${ }^{37}$ Participants were divided into two different groups based on APOE $\varepsilon 4$ status determined by the presence or absence of APOE $\varepsilon 4$ on image and non-imaging phenotypes. ${ }^{37}$ An additional group was also included determined by the presence of $\varepsilon 4$ alleles. ${ }^{37}$ There was significant correlation in clinical diagnosis and cognitive status. Specifically, APOE $\varepsilon 4$ was associated with cognitive performance, temporal lobe atrophy, CSF tau and phosphorylated tau. ${ }^{37}$

\section{Similarities found within neuroimaging}

MRI and PET scans are integral measurements in the diagnostic process for dementia. In particular, MRI is the primary method used to diagnose neurodegenerative diseases. However, PET scans may provide more conclusive results when differentiating and evaluating amyloid beta with comparable biomarkers in recent studies. MRI neuroimaging emerged as the measurement of choice due to the impractical clinical use of carbon-11 Pittsburgh compound-B (C-PIB) radioisotope tracer identified by PET scans. ${ }^{38}$ The onsite cyclotron and specialised radiochemistry infrastructure are required to work in conjunction with C-PIB isotope, ${ }^{38}$ whereas newly developed PET tracers, 18Fflorbetapir (2,3), 18F-florbetaben $(4,5)$ and $18 \mathrm{~F}$-flutemetamol, are radiolabelled with longer lived fluorine-18 isotopes, making PET scans more practical for clinical use..$^{39}$

With the development of these three PET tracers and continued advancements in biomarker research, the diagnostic process is becoming more accurate and easier to conduct. These tracers, despite their vast potential and usability, do have flaws, although small. A study was conducted to find new methods for PET scanning that would compensate for the systematic bias caused by PET scanning's high capacity to take in white and grey matter. ${ }^{39}$ The rising levels of both white and grey matter are proportionate to the fibrillar amyloid beta levels. However, non-specific white matter remains present despite fibrillar amyloid beta loads. ${ }^{39}$ This results in the noticeable development of white matter with differing pattern formations within amyloid beta-positive and amyloid beta-negative images when evaluating scans on standard single-template PET-driven registrations. ${ }^{39} \mathrm{~A}$ fully automated PET-only registration method was developed where a linear combination of the first and second components from a decomposition analysis of 18F-flutemetamol was used to model a synthetic template spanning the entire range of both amyloid beta-positive and amyloid beta-negative. ${ }^{39}$ The new method was proven to be highly successful and allowed for a robust and accurate registration of $18 \mathrm{~F}$-flutemetamol without the need for an MRI. ${ }^{39}$

In this study, 117 participants were analysed. Some were cross-examined in multiple groups when presenting symptoms overlapped. Seventy subjects consisted of 25 cognitively healthy participants; 19 participants with amnesiac mental cognitive impairment (aMCI); 26 participants presented with $\mathrm{AD}$ in the template creation cohort group; 47 patients presented with $14 \mathrm{MCI}, 27 \mathrm{AD}$; and 5 patients exhibited non-AD, with one unspecified case of dementia identified in the registration validation chart group. ${ }^{39}$ The classification was needed to validate method effectuality. The study further negates MRI as the dominant method for scanning initial diagnosis across the broad spectrums of dementia. Although the study is specific to $\mathrm{AD}$, there is potential for future analysis of dementia types and possibly other neurodegenerative diseases in general.

The similarities in dementia type using neuroimaging biomarkers make it incredibly difficult to differentiate between FTD, svPPA, nfvPPA and AD since the subcallosal media prefrontal cortex atrophied into the four types. ${ }^{40}$ Due to the inability of the medial temporal lobe atrophy (MTA) to be evaluated in clinical settings, it is most often neglected as the primary method for diagnosis. ${ }^{41}$ Volumetric analysis, a method heavily used with MTA, provides an 'accurate and detailed measure of a predetermined circumscribed area or region of interest'. ${ }^{41}$ In relation to $\mathrm{AD}$, the hippocampus is the most active part of the brain in this disease and is also the prime location to perform volumetric analysis. ${ }^{41}$ In conjunction with this method's impractical use in clinical applications, it also has a few more drawbacks that stem from manual volumetric analysis. The tracer used to delineate the hippocampus's boundaries, anterior and posterior limits must be trained to do so. ${ }^{41}$ Segmentation of the hippocampus afterwards would take anywhere from 20 to 30 min depending on the user, which signifies this method's widely disadvantageous clinical use despite great results. ${ }^{41}$ Visual assessment tests are adversely viewed due to inaccuracy and lack of precision, but are highly practical for clinical use due to their ability to be performed on several scans simultaneously. More specifically, other studies have shown that the visual assessment of the medial temporal lobes (MTL) rendered similar prediction accuracy in multivariate classification and manual hippocampal volumes. ${ }^{41}$ Other studies have shown that this method failed to detect any patients 
carrying mutations of familial $\mathrm{AD}$ to be at high risk, or detect progression over time, which hinders this discovery's applicability in clinical applications.

To combat the shortcomings found within MTA's usability, a proposed method of diagnosis was introduced to highlight the potential for a new two-dimensional method for measuring MTA through current neuroimaging technology. It should be stated that this method has yet to be tested by clinical researchers, but the vast potential and possible success of this method could push the evaluation of MTA to the forefront of diagnostic methods for dementia. The proposed method focused on the detection of atrophy before symptoms arose and consisted of the measurement of three brain regions on a single MRI slide, where researchers initially took the data from those slides to calculate a simple ratio. ${ }^{41}$ From this initial step, researchers began to manually trace the regions found on the coronal slide at the level of the interpeduncular fossa on the thermal infrared radiation (TIR) sequence through use of the pointer-rule tool found on any visual software used for DITCOM images. ${ }^{41}$ To further expound on the three brain regions evaluated after the initial step, the MTL was defined as the four-sided space boarded in its inferior side by the tentorium cerebelli. ${ }^{41}$ The cerebral peduncles are located on the medial side of the brain-the upper side by the roof of the temporal horn of the lateral ventricle, its lateral side by the collateral sulcus, and a straight line linking the collateral sulcus with the lateral edge of the temporal horn of the lateral ventricle. ${ }^{41}$ The second region evaluated was the parenchyma, which houses the hippocampus and the parahippocampal gyrus, with the exclusion of the fimbria taenia and plexus choroideus in this specific study. ${ }^{41}$ The last region evaluated was the body of the ipsilateral lateral ventricle. ${ }^{41}$

Tau and phosphorylated tau P-tau181P are known for delivering positive outcomes when diagnosing different types of dementia, most notably AD. Applying neuroimaging techniques such as PET scans to these specific biomarkers is crucial in diagnosing neurodegenerative diseases in general and assessing the burden of tau in clinical trials for antidementia drugs. ${ }^{42}$ Tau PET was considered potentially useful for antemortem assessment of non-AD tauopathies, such as progressive supranuclear palsy (PSP), corticobasal degeneration (CBD) and some variants of FTD. ${ }^{42} 43$ [18F]THK5351 was a part of the first generation of tau PET tracers purposed to detect tau aggregates in the form of paired helical filaments tau (PHF-tau) in AD. ${ }^{44}$ Studies surrounding this PET tracer found there was prominent retention in patients with PSP and CBD within the midbrain and the basal ganglia. The pathology is indicative of disease progression due to the positive correlation with the clinical severity of PSP caused by the amount of tracer retention. ${ }^{44}$ With regard to $[18 \mathrm{~F}]$ THK5351 binding mechanics, it was found to correlate significantly with monoamine oxidase-B (MAO-B) density and tau levels, indicating that [18F]THK5351 reflects tau pathology and reactive astrocytes in $\mathrm{AD}$ brains. ${ }^{45}$
The latest advancements and studies of tau PET pharmaceuticals, specifically [18F]AV1451, pinpointed a significant difference between control subjects and those with $\mathrm{AD} .{ }^{46}$ For studies involving [18F]AV1451, results delineated elevated tracer retention where tau pathology was heavily observed in PSP and CBD. ${ }^{42}$ Discrepancies were mentioned in several studies that categorised differences between antemortem PET and postmortem in vitro binding research of non-AD tauopathies. ${ }^{42}$ Moreover, [18F]AV1451 failed to bind to four-repeat tau lesions in PSP and CBD in in vitro autoradiography validation studies. $^{47}$

Through recent cross-sectional PET studies, APOE $\varepsilon 4$ was found to have abnormally low measurements of the cerebral metabolic rate for glucose (CMRgl) located in the same regions found in patients with $\mathrm{AD} .{ }^{36}{ }^{40} \mathrm{In}$ a study aimed to characterise longitudinal CMRgl declines in normal $\varepsilon 4$ heterozygotes, the potential to check the efficacy of current treatment methods and potential candidate therapies for $\mathrm{AD}$ prevention were estimated. Researchers considered how this paradigm could efficiently be used to test the potential of $\mathrm{AD}$ preventative therapies. ${ }^{36}$ Researchers identified several limitations in using PET measurements and other biological markers in normal APOE $\varepsilon 4$ carriers to test the potential of preventive candidate treatments for cognitive AD-related impairment. Moreover, preventative studies in $\varepsilon 4$ carriers may not be generalised to $\varepsilon 4$ non-carriers between positive and negative findings. ${ }^{36}$ There is also the issue of PET CMRgl attenuation in CMRgl decline. This attenuation occurs before clinical symptoms appear and is associated with a decreased risk of AD symptoms. ${ }^{36}$ However, APOE genotypes and PET measurements have not proven through clinical analysis a patient's probability of developing AD symptoms. ${ }^{36}$ Due to limited research, preventative therapies do not accurately predict or outweigh the psychological or social risk that would come from possibly misdiagnosing a patient. ${ }^{36}$ Despite these hindrances, the study produced a paradigm for characterising the potential of preventative $\mathrm{AD}$ treatments without evaluating countless studies and clinical trials.

The APOE $\varepsilon 4$ heterozygotes measured had significant CMRgl declines in the vicinity of temporal, posterior cingulate, prefrontal cortex, basal forebrain, parahippocampal gyrus and thalamus which proved to be greater in the $\varepsilon 4$ non-carriers. ${ }^{36}$ The two main groups of participants consisted of $10 \varepsilon 4$ heterozygotes which included 7 women and 3 men. All presenting with the $\varepsilon 3 / \varepsilon 4$ genotype were $55.9 \pm 3.4$ years of age $($ mean \pm SD) and had $15.4 \pm 2.9$ years of education. ${ }^{36}$ The $\varepsilon 4$ non-carriers included 10 women and 5 men. Ten with the $\varepsilon 3 /$ $\varepsilon 3$ genotype and five with the $\varepsilon 2 / \varepsilon 3$ genotype were $57.1 \pm 4.4$ years of age and had $16.1 \pm 1.9$ years of education. ${ }^{36}$ In an attempt to test a candidate's preventative treatment potential for developing AD symptoms, researchers estimated the results of 50-115 cognitively healthy $\varepsilon 4$ heterozygotes between 50 and 63 years of age per active group. The placebo treatment group needed 
to detect a $25 \%$ attenuation in CMRgl decline in the temporal cortex, posterior cingulate cortex, basal forebrain, parahippocampal/lingual gyri and thalamus at $80 \%$ power over 2 years. ${ }^{36}$ If there was a linear digression of CMRg1, then the same number of cognitively normal $\varepsilon 4$ heterozygotes per active and placebo treatment group would be necessary to detect a $50 \%$ attenuation in CMRgl declines with $80 \%$ power. ${ }^{36}$ It was also estimated that 207-289 cognitively healthy $\varepsilon 4$ non-carriers from ages 50 to 63 per active and placebo treatment group were needed to detect a $25 \%$ attenuation in age-related CMRgl declines in the anterior cingulate cortex, parietal cortex, posterior cingulate cortex and the caudate nucleus at $80 \%$ power. ${ }^{36}$ From these estimations, as well as in previous findings from other studies, regional CMRg1 in $\varepsilon 4$ heterozygotes continued to decline in the temporal lobes, posterior cingulate, prefrontal, basal forebrain, parahippocampal/lingual gyri and thalamus during the 2-year interval between baseline and follow-up scans.

\section{DISCUSSION}

\section{Main findings}

Evidence suggests neuroimaging with amyloid PET scanning and newly found pet tracers (18Fflorbetapir [2,3], 18F-florbetaben [4,5] and 18F-flutemetamol) is more effective in diagnosing Alzheimer's and aMCI, and has vast potential to detect other neurodegenerative diseases. A fully automated, newly developed PET-only registration method using $18 \mathrm{~F}$-flutemetamol was used to model a synthetic template spanning the entire range of both amyloid beta-positive and amyloid beta-negative. ${ }^{39}$ The new method was proven to be highly successful and allowed for a robust and accurate registration of 18F-flutemetamol without the need for an MRI. ${ }^{39}$ The use of miRNA dysregulation appears to be an effective new method developed for use when diagnosing the four different types of dementia. Further evaluation of the dysregulation of miRNA expression in the peripheral blood may serve as a potent source for diagnosing $\mathrm{AD}$ and other neurodegenerative diseases. ${ }^{17}{ }^{18}$ Some researchers investigating solely on MHPG levels in CSF and serum monoamines found CSF and serum MHPG to be the most valuable markers to differentiate $\mathrm{AD}$ from DLB and PD. ${ }^{4}$ As for annexin A5 levels evaluated between test subjects and control, the plasma levels were much higher in $\mathrm{AD}$-affected mice than in the control group, ${ }^{13} 30$ suggesting annexin A5 may be an effective biomarker for $\mathrm{AD}$ and has been proven to be an effective biomarker in DLB. ${ }^{2} 32$

A new method introduced to combat the shortcomings found within MTA's usability focused on the detection of atrophy before symptoms arose and consisted of the measurement of three brain regions on a single MRI slide. ${ }^{41}$ This method has not been clinically tested to date, but has vast potential. Researchers identified several limitations using PET measurements and other biological markers in normal APOE $\varepsilon 4$ carriers to test the potential of preventive candidate treatments for cognitive AD-related impairment. Furthermore, preventative studies in $\varepsilon 4$ carriers may not be generalised to $\varepsilon 4$ non-carriers between positive and negative findings. ${ }^{36}$

\section{Limitations}

Limitations were discovered before and during the course of this research. Available articles and methods accessed from research databases were limited to the availability of studies on the topic meeting inclusion criteria. However, there was one factor of influence that could not be controlled by man, but can place restrictions on methodology. Lastly, the aptitude of the researchers writing these varying articles was not cited effectively in their written works, which raised concern regarding authenticity and credibility.

Understanding the limitations and negatives in testing CSF is extremely important in order for innovation and creativity to develop a future reliance on other methods for diagnosing different types of dementia. From the studies highlighted, there were several new methods that stood out more than others, more specifically changing the initial belief that conducting MRIs is the best neuroimaging method for diagnosing dementia and indicating PET scans are considerably more accurate in discerning dementia types than originally believed. Other studies were hindered by the shortcomings of PET scans, but by using newly developed isotopes and PET tracers (18Fflorbetapir [2,3], 18F-florbetaben [4,5], 18F-flutemetamol and [18F]AFV1451) that were shown to be more reliable and clinically friendly for avid use. ${ }^{394}$ Overall, some studies were either shedding light on PET scans' shortcomings within studies, or highlighting the potential of PET scans to be used in more clinical settings by using $18 \mathrm{~F}$-flutemetamol to model a synthetic template. The results from one specific study were able to show that an MRI was not required for an accurate registration of $18 \mathrm{~F}$-flutemetamol. ${ }^{39}$ Another PET scanning study had highlighted a few key issues with PET scans and still managed to find that regional CMRg1 in $\varepsilon 4$ heterozygotes continued to decline in the temporal lobes, posterior cingulate, prefrontal, basal forebrain, parahippocampal/lingual gyri and the thalamus during the 2-year interval between their baseline and follow-up scans. ${ }^{36}$ Regarding the PET tracer $[18 \mathrm{~F}]$ THK5351 binding mechanics, it was found to correlate significantly with MAO-B density and tau levels, indicating $[18 \mathrm{~F}]$ THK5351 reflects tau pathology and reactive astrocytes in $\mathrm{AD}$ brains, which again shows the importance of PET neuroimaging. ${ }^{45}$

\section{Implications}

The initial study evaluated the effectiveness of 18Fflorbetapir (2,3), 18F-florbetaben $(4,5)$, 18F-flutemetamol and [18F]AFV145. It indicated great potential for what could be accomplished when compared with its predecessor. In the future, I would like to use these 
newly found PET tracers to retest and re-evaluate the studies conducted that used amyloid PET scanning and documented issues discerning dementia types, thus making the claims and results of those other studies more credible. Regarding limitations for creating this systematic review, there were no particular limitations apart from obtaining access to key articles from selective databases. All of the studies referenced provided significant evidence for new methods and biomarkers. By solely focusing on MHPG levels in CSF and serum monoamines, researchers discovered that CSF and serum MHPG were the most valuable markers to differentiate AD from DLB and PD. ${ }^{4}$ In relation to discovering the importance of serum MHPG, another study discovered significant associations between APOE $\varepsilon 4$ and cognitive performance, temporal lobe atrophy, CSF tau, and phosphorylated tau. ${ }^{37}$ There were other studies that found different results after conducting research. More specifically, there have been studies that found the visual assessment of the MTL having similar predictive accuracy when compared with multivariate classification and manual hippocampal volumes. ${ }^{41}$ Other studies, however, have shown this method failed to detect patients carrying mutations of familial $\mathrm{AD}$ to be at high risk or detect progression of the disease, which may hinder this discovery's applicability in clinical applications.

\section{Contributors JMAM was the sole writer. TW and SX reviewed and revised the paper.}

Funding This study was funded by the Municipal Human Resources Development Program for Outstanding Leaders in Medical Discipline in Shanghai (2017BR054), Shanghai Jiao Tong University School of Medicine Collaborative Innovation Project (TM201728), Shanghai Municipal Education Commission-Gaofeng Clinical Medicine Grant Support (20172029) and also the National Natural Science Foundation of China (81571298).

Competing interests None declared.

Patient consent for publication Not required.

Provenance and peer review Not commissioned; externally peer reviewed.

Data sharing statement № additional data are available.

Open access This is an open access article distributed in accordance with the Creative Commons Attribution Non Commercial (CC BY-NC 4.0) license, which permits others to distribute, remix, adapt, build upon this work non-commercially, and license their derivative works on different terms, provided the original work is properly cited and the use is non-commercial. See: http://creativecommons.org/ licenses/by-nc/4.0

\section{REFERENCES}

1. Mayeux R. Biomarkers: potential uses and limitations. NeuroRx 2004:1:182-8.

2. Menéndez-González M. Biomarkers in neurodegenerative disorders: translating research into clinical practice. Frontiers in Aging Neuroscience 2014;6.

3. Naylor S. Biomarkers: current perspectives and future prospects. Expert Review of Molecular Diagnostics 2003;3:525-9.

4. Janssens J, Vermeiren Y, Fransen E, et al. Cerebrospinal fluid and serum MHPG improve Alzheimer's disease versus dementia with Lewy bodies differential diagnosis. Alzheimer's \& Dementia: Diagnosis, Assessment \& Disease Monitoring 2018;10:172-81.

5. Menéndez-González M. Routine lumbar puncture for the early diagnosis of Alzheimer's disease. Is it safe? Frontiers in Aging Neuroscience 2014;6.
6 Parnetti L, Farotti L, Eusebi P, et al. Differential role of CSF alphasynuclein species, tau, and A $\beta 42$ in Parkinson's disease. Front Aging Neurosci 2014;6.

7. Shang J, Yamashita T, Fukui Y, et al. Different associations of plasma biomarkers in Alzheimer's disease, mild cognitive impairment, vascular dementia, and ischemic stroke. J Clin Neurol 2018;14.

8. Gomez-Ramirez J, Wu J. Network-based biomarkers in Alzheimer's disease: review and future directions. Front Aging Neurosci 2014;6.

9. Hanagasi HA, Bilgiç B, Emre M. Neuroimaging, biomarkers, and management of dementia with Lewy bodies. Front Neurol 2013;4.

10. Bott NT, Radke A, Stephens ML, et al. Frontotemporal dementia: diagnosis, deficits and management. Neurodegener Dis Manag 2014;4:439-54.

11. Irwin DJ, Trojanowski JQ, Grossman M. Cerebrospinal fluid biomarkers for differentiation of frontotemporal lobar degeneration from Alzheimer's disease. Front Aging Neurosci 2013;5.

12. Kurz A, Kurz C, Ellis $\mathrm{K}$, et al. What is frontotemporal dementia? Maturitas 2014;79:216-9.

13. Yamaguchi M, Kokai Y, Imai S-I, et al. Investigation of annexin A5 as a biomarker for Alzheimer's disease using neuronal cell culture and mouse model. J Neurosci Res 2010;3.

14. Young JJ, Lavakumar M, Tampi D, et al. Frontotemporal dementia: latest evidence and clinical implications. Ther Adv Psychopharmacol 2018;8:33-48.

15 Jacobsen SR, ed. Vascular dementia: risk factors, diagnosis, and treatment. Hauppauge, NY: Nova Science, 2011.

16. Duara $R$, Loewenstein $D A$, Shen $Q$, et al. The utility of age-specific cut-offs for visual rating of medial temporal atrophy in classifying Alzheimer's disease, $\mathrm{MCl}$ and cognitively normal elderly subjects. Front Aging Neurosci 2013;5.

17. Schipper HM, Maes OC, Chertkow HM, et al. MicroRNA expression in Alzheimer blood mononuclear cells. Gene Regul Syst Bio 2007;1:GRSB.S361-274.

18. Dong $\mathrm{H}, \mathrm{Li} J$, Huang $\mathrm{L}$, et al. Serum MicroRNA Profiles Serve as Novel Biomarkers for the Diagnosis of Alzheimer's Disease. Dis Markers 2015;2015:1-11.

19. Aliev G, Gasimov E, Obrenovich ME, et al. Atherosclerotic lesions and mitochondria DNA deletions in brain microvessels: implication in the pathogenesis of Alzheimer's disease. Vasc Health Risk Manag 2008;4:721-30.

20. Aliev G, Palacios HH, Gasimov E, et al. Oxidative stress induced mitochondrial failure and vascular hypoperfusion as a key initiator for the development of Alzheimer disease. Pharmaceuticals 2010;3:158-87.

21. Sharma N. Exploring Biomarkers for Alzheimer's Disease. Journal Of Clinical And Diagnostic Research 2016.

22 Charney DS, Nestler EJ, eds. Neurobiology of mental illness. 2nd ed. Oxford New York: Oxford University Press, 2004.

23. Gaengel K, Genové G, Armulik A, et al. Endothelial-mural cell signaling in vascular development and angiogenesis. Arterioscler Thromb Vasc Biol 2009;29:630-8.

24. Lakatos A, Goldberg NR, Blurton-Jones M. Integrated analysis of genetic, behavioral, and biochemical data implicates neural stem cell-induced changes in immunity, neurotransmission and mitochondrial function in dementia with Lewy body mice. Acta Neuropathol Commun 2017;5

25. Lin $\mathrm{C}-\mathrm{H}$, Yang $\mathrm{S}-\mathrm{Y}$, Horng $\mathrm{H}-\mathrm{E}$, et al. Plasma Biomarkers Differentiate Parkinson's Disease From Atypical Parkinsonism Syndromes. Front Aging Neurosci 2018;10.

26. Swanson D, Block R, Mousa SA. Omega-3 fatty acids EPA and DHA health benefits throughout life. Adv Nutr 2012;3:1-7.

27. Yates KF, Sweat V, Yau PL, et al. Impact of metabolic syndrome on cognition and brain: a selected review of the literature. Arterioscler Thromb Vasc Biol 2012;32:2060-7.

28. $\mathrm{Yu}$ X-H, Fu Y-C, Zhang D-W, et al. Foam cells in atherosclerosis. Clinica Chimica Acta 2013;424:245-52.

29. Ross R, Agius L. The process of atherogenesis--cellular and molecular interaction: from experimental animal models to humans. Diabetologia 1992;35 Suppl 2:S34-S40.

30. Sohma H, Imai S, Takei N, et al. Evaluation of annexin A5 as a biomarker for Alzheimer's disease and dementia with Lewy bodies. Front Aging Neurosci 2013;5.

31. Liu P, Reichl JH, Rao ER, et al. Quantitative comparison of densecore amyloid plaque accumulation in amyloid- $\beta$ protein precursor transgenic mice. J Alzheimers Dis 2017;56:743-61.

32. Pereira JM, Acosta-Cabronero J, Pengas G, et al. VBM with viscous fluid registration of gray matter segments in SPM. Front Aging Neurosci 2013;5.

33. Bedrood S, Jayasinghe S, Sieburth D, et al. Annexin A5 directly interacts with amyloidogenic proteins and reduces their toxicity. Biochemistry 2009;48:10568-76. 
34. Ludtmann MHR, Angelova PR, Horrocks MH, et al. $\alpha$-synuclein oligomers interact with ATP synthase and open the permeability transition pore in Parkinson's disease. Nat Commun 2018;9.

35. Renehan AG, Booth C, Potten CS. What is apoptosis, and why is it important? BMJ 2001;322:1536-8.

36 Reiman EM, Caselli RJ, Chen K, et al. Declining brain activity in cognitively normal apolipoprotein E4 heterozygotes: A foundation for using positron emission tomography to efficiently test treatments to prevent Alzheimer's disease. Proceedings of the National Academy of Sciences 2001;98:3334-9.

37. Risacher SL, Kim S, Shen L, et al. The role of apolipoprotein E (ApoE) Genotype in early mild cognitive impairment (E-MCI). Front Aging Neurosci 2013;5

38. Koedam EL, van der Vlies AE, van der Flier WM, et al. Cognitive correlates of cerebrospinal fluid biomarkers in frontotemporal dementia. Alzheimers Dement 2013;9:269-75.

39. Lilja J, Leuzy A, Chiotis K, et al. Spatial normalization of [ $\left.{ }^{18} \mathrm{~F}\right]$ flutemetamol PET images utilizing an adaptive principal components template. Journal of Nuclear Medicine 2018;118.

40. Lindberg O, Westman E, Karlsson S, et al. Is the subcallosal medial prefrontal cortex a common site of atrophy in Alzheimer's disease and frontotemporal lobar degeneration? Front Aging Neurosci 2012;4.
41. Menéndez-González M, López-Muñiz A, Vega JA, et al. MTA index: a simple 2D-method for assessing atrophy of the medial temporal lobe using clinically available neuroimaging. Frontiers in Aging Neuroscience 2014;6.

42. Ishiki A, Harada R, Kai H, et al. Neuroimaging-pathological correlations of $\left[{ }^{18} \mathrm{~F}\right] \mathrm{THK} 5351 \mathrm{PET}$ in progressive supranuclear palsy. Acta Neuropathol Commun 2018;6.

43. Villemagne VL, Doré V, Burnham SC, et al. Imaging tau and amyloid- $\beta$ proteinopathies in Alzheimer disease and other conditions. Nat Rev Neurol 2018;14:225-36.

44. Harada R, Okamura N, Furumoto S, et al. 18F-THK5351: a novel PET radiotracer for imaging neurofibrillary pathology in Alzheimer disease. J Nucl Med 2016;57:208-14.

45. Harada R, Ishiki A, Kai H, et al. Correlations of ${ }^{18} \mathrm{~F}-\mathrm{THK} 5351$ PET with Postmortem Burden of Tau and Astrogliosis in Alzheimer Disease. J Nucl Med 2018;59:671-4.

46. Johnson KA, Schultz A, Betensky RA, et al. Tau positron emission tomographic imaging in aging and early Alzheimer disease. Ann Neurol 2016;79:110-9.

47. Marquié M, Normandin MD, Meltzer AC, et al. Pathological correlations of [F-18]-AV-1451 imaging in non-alzheimer tauopathies. Annals of Neurology 2017;81:117-28.



Joshua Marvin Anthony Maclin, an undergraduate neuroscience major attending Earlham College in Richmond, IN, worked in the Geriatric Psychiatry department of the Shanghai Mental Health Center in 2018 as a visiting student. He also volunteers, as a Bonner Scholar, at Richmond State Hospital and Reid Health Hospital as a lab volunteer. In conjunction with Bonner, he has recently been accepted into the McNair Scholar program where he will continue to conduct research of neurodegenerative diseases, while also pursuing his future aspirations of attending medical school at the Vagelos College of Physicians and Surgeons at Columbia University. 